\title{
Viel Arbeit für weniger Arbeit
}

\author{
Stefan Schneider, Matthias Winistörfer, Rosmarie Glauser
}

Stichtag ist der 1. Januar 2005. Dann unterstehen alle Assistenzärztinnen und -ärzte dem Arbeitsgesetz. Maximale Arbeitszeit und weitere Arbeitsbedingungen sind verbindlich geregelt. Für die betroffenen Arbeitgeber bringt dies initial ein Mehr an Aufwand und Kosten. Eine vorausschauende und sachdienliche Planung vermag Probleme zu reduzieren.

\section{Vorgeschichte}

Die überlangen Arbeitszeiten der Assistenz- und Oberärztinnen und -ärzte sind seit Jahrzehnten ein Thema. In den achtziger Jahren konnten erste Erfolge zur Begrenzung der Arbeitszeit erzielt werden, z. B. mit einer Verordnung für die kantonalbernischen Spitäler, die eine maximale Wochenarbeitszeit von 58 Stunden vorsah; diese war allerdings sehr vage formuliert, und die Einhaltung wurde bis 1998 nicht überprüft. Ende neunziger Jahre intensivierte sich die Diskussion erneut, als die breite Öffentlichkeit über die Arbeitszeitmissstände informiert wurde. Der Bleistiftstreik von 1998 im Kanton Zürich und andere Aktionen führten schliesslich zu Verordnungen und Gesamtarbeitsverträgen mit einer wöchentlichen Höchstarbeitszeit von 50 Stunden.

Immer deutlicher zeigte sich aber, dass eine gesamtschweizerisch verbindliche Lösung gefunden werden musste, um überall in der Schweiz einen Minimalstandard bezüglich Arbeitszeiten zu schaffen. Die Idee, eine Änderung des Arbeitsgesetzes anzustreben, entstand 1997. Nationalrat Marc F. Suter reichte schliesslich 1998 eine parlamentarische Initiative ein, die die Unterstellung der Assistenzärztinnen und -ärzte unter das Arbeitsgesetz forderte. Am 22. März 2002 nahmen die Räte die entsprechende Änderung des Arbeitsgesetzes in der Schlussabstimmung an. Die Assistenzärztinnen und -ärzte unterstehen somit ab 2005 dem Arbeitsgesetz.

\section{Vorgaben des Arbeitsgesetzes}

Korrespondenz:

VSAO-ASMAC

Dählhölzliweg 3

Postfach 229

CH-3000 Bern 6
- Die wöchentliche Höchstarbeitszeit beträgt 50 Stunden.

- Als Arbeitszeit gilt die Zeit, während der sich der Arbeitnehmer zur Verfügung des Arbeitgebers zu halten hat.

- Die tägliche Ruhezeit beträgt mindestens 11 aufeinanderfolgende Stunden. Sie darf mehrmals pro Woche auf 9 Stunden herabgesetzt werden, sofern die Dauer von 12 Stunden im Durchschnitt von 2 Wochen eingehalten wird.

- Im Zeitraum von 4 Wochen darf ein Arbeitnehmer an höchstens 7 Tagen auf Pikett sein oder Piketteinsätze leisten. Pikettdienst im Betrieb gilt voll als Arbeitszeit.

- Sonntagsarbeit von einer Dauer bis zu 5 Stunden ist durch Freizeit auszugleichen. Dauert sie länger als 5 Stunden, so ist während der vorhergehenden oder der nachfolgenden Woche im Anschluss an die tägliche Ruhezeit ein Ersatzruhetag von mindestens 24 aufeinanderfolgenden Stunden zu gewähren.

- In Spitälern darf die Nachtarbeit gemäss Globalbewilligung 12 Stunden innert eines Zeitraumes von 12 Stunden betragen, wenn ein grosser Teil davon reine Präsenzzeit darstellt, eine Ruhegelegenheit vorhanden ist und die Ruhezeit mindestens 4 Stunden umfasst. Die Pausen gelten als Arbeitszeit. Den Arbeitnehmern ist eine tägliche Ruhezeit von 12 Stunden zu gewähren.

- Für Nachtarbeit zwischen 23.00 und 6.00 Uhr müssen Zeitzuschläge gewährt werden. Bei finanzieller Vergütung von Überzeit werden Überzeitzuschläge geschuldet.

\section{Forderungen zur Umsetzung}

Bisher konnten Assistenzärztinnen und -ärzte zeitlich fast unlimitiert eingesetzt werden, und dies oft erst noch ohne Kostenfolge für die Arbeitgeber. Wöchentliche Arbeitszeiten von 65 bis 70 Stunden sind deshalb noch immer anzutreffen, trotz der nachgewiesenermassen deletären Auswirkungen auf die Qualität der Dienstleistung, die Fehlerhäufigkeit, die Produktivität sowie die Gesundheit der Mitarbeitenden. Die Unterstellung der Assistenzärztinnen und -ärzte unter das Arbeitsgesetz führt deshalb in vielen 
Betrieben zwingend zu einer Reduktion der wöchentlichen Arbeitszeit und Umstellungen der Dienstsysteme, wahrscheinlich sogar $\mathrm{zu}$ einem eigentlichen Paradigmenwechsel beim Einsatz der Ärztinnen und Ärzte. Um dies zu erreichen, wird es nicht genügen, einige neue Stellen zu schaffen. Vielmehr ist eine grundlegende Reorganisation der ärztlichen und teilweise nichtärztlichen Arbeitsprozesse und der täglich gelebten Weiterbildung im Spital nötig. An einigen Spitälern konnten in den letzten Jahren im Rahmen von Arbeitszeitreduktionen aufgrund von Gesamtarbeitsverträgen bereits Erfahrungen mit solchen Projekten gesammelt werden. Überall fand sich einerseits ein beachtliches Potential an betrieblichen Verbesserungsmöglichkeiten, andererseits stiessen die Verantwortlichen aber auch auf unerwartet grosse Schwierigkeiten. Generell gilt, dass Reorganisationsprozesse Zeit brauchen und fast immer mit (Investitions-) Kosten verbunden sind.

\section{Lösungsansätze}

Effiziente und effektive Arbeitsprozesse erfordern eine klare Regelung der Zuständigkeiten und Kompetenzen und des Informationsflusses. Tagesabläufe und Schichtpläne sind unter dem Gesichtspunkt der Arbeitszeiten der Assistenzärztinnen und -ärzte zu überprüfen und gegebenenfalls zu optimieren. Informatikmittel wie die elektronische Krankengeschichte und Planungssysteme können heute Arbeitsprozesse vereinfachen. Andere Aufgaben (z. B. ICD-10-Codierung und allgemeine Sekretariatsarbeiten), die nicht als Kernkompetenzen von Ärztinnen und Ärzten gelten, sind konsequent an andere Berufsgruppen zu übertragen. Um eine Schaffung von zusätzlichen Stellen wird man kaum herumkommen, wobei es dafür keinesfalls ausschliesslich Ärztinnen und Ärzte braucht. Wenn sich alle Betroffenen bei diesen Veränderungsprozessen direkt einbringen können, werden potentielle Konflikte früh erkannt oder sogar vermieden.

Die Weiterbildung der Assistenzärztinnen und -ärzte darf dabei nicht vergessen gehen. Die Ärzteschaft, also FMH-Fachgesellschaften wie auch der VSAO, und die Spitäler als Weiterbildungsstätten sind gleichermassen gefordert, zukünftig strukturierte Weiterbildungsprogramme $\mathrm{zu}$ entwickeln und auch umzusetzen.
Fachtagung zum revidierten Arbeitsgesetz 4. November 2003, Kursaal Bern, 9.30-16.30 Uhr

Patronatsorganisationen: FMH, seco (Staatssekretariat für Wirtschaft)

Ziele der Fachtagung

Die Teilnehmerinnen und Teilnehmer der Fachtagung ...

- kennen die wesentlichen Änderungen im revidierten Arbeitsgesetz;

- können die Auswirkungen auf ihre Organisation abschätzen;

- kennen ihre Aufgaben und Pflichten in bezug auf die Arbeitszeit;

- erhalten wertvolle Hinweise, wohin sie sich bei Fragen wenden können;

- sind fähig, das revidierte Arbeitsgesetz in ihrer Organisation erfolgreich umzusetzen;

- haben die Möglichkeit, sich mit anderen Teilnehmerinnen und Teilnehmern auszutauschen und von Institutionen zu profitieren, die sich mit der Umsetzung bereits intensiv auseinandergesetzt haben.

Zielgruppen

- Direktorinnen und Direktoren von Spitälern und Kliniken;

- Personalverantwortliche in Spitälern und Kliniken;

- vorgesetzte Personen von Assistenz- und Oberärztinnen und -ärzten;

- Assistenz- und Oberärztinnen und -ärzte;

- weitere an diesem Thema interessierte Personen.

Kosten

Fr. 290.- bzw. Fr. 190.- für VSAO- und/oder FMH-Mitglieder, Fr. 50.- für Studierende.

Informationen und Anmeldung

Verband Schweizerischer Assistenz- und Oberärztinnen und -ärzte, Frau N. Reinmann, Dählhölzliweg 3, Postfach 229, 3000 Bern 6, Tel. 03135044 88, Fax 03135044 89, E-Mail: reinmann@vsao.ch 